\title{
CHARACTERIZATION OF AUTOMORPHISMS ON THE BARRETT AND THE DIEDERICH-FORNAESS WORM DOMAINS
}

\author{
SO-CHIN CHEN
}

\begin{abstract}
In this paper we show that every automorphism on either the Barrett or the Diederich-Fornaess worm domains is given by a rotation in $w$-variable. In particular, any automorphism on either one of these two domains can be extended smoothly up to the boundary.
\end{abstract}

\section{INTRODUCTION}

In several complex variables extending a biholomorphism or an automorphism smoothly up to the boundary is always a very important and fundamental problem which is closely related to the classification problem of domains in $\mathbf{C}^{n}$. The extension phenomenon in general is false as shown in Barrett [3] if the domains are sitting in some general complex manifolds. However, it is still widely believed that such extension phenomena should hold if the domains are contained in $\mathbf{C}^{n}$, namely, we conjecture the following two statements,

Any biholomorphism between two smoothly bounded domains

$D_{1}$ and $D_{2}$ in $\mathbf{C}^{n}, n \geq 2$, can be extended smoothly to a $C R$-diffeomorphism between $\overline{D_{1}}$ and $\overline{D_{2}}$,

and its weaker counterpart

$$
\begin{aligned}
& \text { Any automorphism of a smoothly bounded domain } D \text { in } \mathbf{C}^{n}, \\
& n \geq 2 \text {, can be extended smoothly up to the boundary, i.e., } \\
& \operatorname{Aut}(D)=\operatorname{Aut}(\bar{D}) \text {. }
\end{aligned}
$$

Indeed, it has been shown in Bell and Ligocka [6] that if condition $R$ holds on both $D_{1}$ and $D_{2}$, then (1.1) is valid. Here condition $R$ means that the Bergman projection associated with the domain $D$ maps $C^{\infty}(\bar{D})$ continuously into itself. Condition $R$ was shown to hold on a large class of (pseudoconvex or nonpseudoconvex) domains. But surprisingly Barrett constructed in [1] a smoothly bounded nonpseudoconvex domain $\Omega$ in $\mathbf{C}^{2}$ which fails to satisfy condition $R$.

On the other hand, Diederich and Fornaess in [8] constructed a smoothly bounded pseudoconvex domain $\Omega_{r}$ in $\mathbf{C}^{2}$ which possesses many pathological properties that include a nontrivial Nebenhulle and the nonexistence of a $C^{3}$

Received by the editors April 29, 1991.

1980 Mathematics Subject Classification (1985 Revision). Primary 32A07, 32M05.

Key words and phrases. Automorphism group, Barrett's domain, Diederich-Fornaess domain.

Research partially supported by a FRAP grant from SUNY at Albany. 
plurisubharmonic defining function for $\Omega_{r}$. Very recently Barrett showed in [4] that the Bergman projection associated with $\Omega_{r}$ does not preserve the Sobolev space $W^{k}\left(\boldsymbol{\Omega}_{r}\right)$ if $k \in \mathbf{R}$ is large enough. It is still not clear whether condition $R$ holds on $\Omega_{r}$ or not.

In this article we want to show that despite these pathological properties found on $\Omega$ and $\Omega_{r}$, statement (1.2) is still valid on both $\Omega$ and $\Omega_{r}$. In fact we can prove more, namely,

Main Theorem. Any automorphism $f$ of either the Barrett or the DiederichFornaess worm domains is given by a rotation in $w$-variable, i.e., $f(z, w)=$ $\left(z, e^{i \phi} w\right)$ for some constant $\phi \in \mathbf{R}$. In particular, $f$ can be extended smoothly up to the boundary.

We make a remark here that although $\Omega$ does not satisfy condition $R$, it still enjoys an a priori estimate on Sobolev space $W^{k}(\Omega)$ as shown in Boas and Straube [7].

\section{Proof ON THE BarRetT's Domain}

We first recall the definition of $\Omega$. The domain $\Omega$ is a smoothly bounded domain defined in $\mathbf{C}^{2}$ as follows,

$$
\Omega=\left\{(z, w) \in \mathbf{C}^{2}|1<| w|<6,| z-c(|w|) \mid>r_{1}(|w|) \text { and }|z|<r_{2}(|w|)\right\},
$$

where the functions $r_{1}(|w|), r_{2}(|w|)$, and $c(|w|)$ are chosen to meet the following conditions: Let $k$ be a positive integer. Define

$$
\begin{aligned}
& r_{1}(x)= \begin{cases}3-\sqrt{x-1} & \text { for } x \text { near } 1, \\
\text { decreasing } & \text { for } x \in[1,2], \\
1 & \text { for } x \in[2,5], \\
\text { increasing } & \text { for } x \in[5,6], \\
3-\sqrt{6-x} & \text { for } x \text { near } 6 .\end{cases} \\
& r_{2}(x)= \begin{cases}3+\sqrt{x-1} & \text { for } x \text { near } 1, \\
\text { increasing } & \text { for } x \in[1,2], \\
4 & \text { for } x \in[2,5], \\
\text { decreasing } & \text { for } x \in[5,6], \\
3-\sqrt{6-x} & \text { for } x \text { near } 6 .\end{cases}
\end{aligned}
$$

and

$$
c(x)= \begin{cases}0 & \text { for } x \in[1,2], \\ \text { decreasing } & \text { for } x \in[2,3], \\ (x-3)^{2 k}-1 & \text { for } x \text { near } 3, \\ \text { increasing } & \text { for } x \in[3,4], \\ -(x-4)^{2 k}+1 & \text { for } x \text { near } 4, \\ \text { decreasing } & \text { for } x \in[4,5], \\ 0 & \text { for } x \in[5,6] .\end{cases}
$$

Then the following theorem that shows $\Omega$ fails to satisfy condition $R$ was proved in Barrett [1]. 
Theorem. $P\left(C_{0}^{\infty}(\Omega)\right)$ is not contained in $L^{p}(\Omega)$ for $p \geq 2+\frac{1}{k}$, where $P$ is the Bergman projection associated with $\Omega$.

Now we proceed to prove our main theorem on this domain. Let $f=\left(f_{1}, f_{2}\right)$ be an automorphism of $\Omega$. We first show the following lemma. A similar statement was proved in Boas and Straube [7].

Lemma 2.1. Let $g$ be a bounded holomorphic function on $\Omega$. Then $g$ can be extended holomorphically to $D$, where

$$
D=\left\{(z, w) \in \mathbf{C}^{2}|| z \mid<r_{2}(|w|) \text { and } 1<|w|<6\right\} .
$$

Proof. By Laurent series expansion one can write

$$
g(z, w)=\sum_{n=-\infty}^{\infty} a_{n}(z) w^{n},
$$

where

$$
a_{n}(z)=\frac{1}{2 \pi i} \int_{|w|=R} \frac{g(z, w)}{w^{n+1}} d w,
$$

and $|w|=R$ is any circle contained in the slice corresponding to $z$. Then one can see easily from $(2.3)$ that $a_{n}(z)$ is locally uniformly bounded and can be extended to a holomorphic function on $\Delta(0 ; 4)$; i.e.,

$$
a_{n}(z) \in H(\Delta(0 ; 4)) \text { for all } n \in \mathbf{Z} \text {. }
$$

Next we show as in [7] that the series (2.2) in fact converges on $\{(z, w) \in$ $\mathbf{C}^{2}|| z \mid<3$ and $\left.1<|w|<6\right\}$ to a holomorphic function. Consider first the nonnegative indices, i.e.,

$$
\sum_{n=0}^{\infty} a_{n}(z) w^{n}
$$

Put $u(z)=\varlimsup_{n \rightarrow \infty}\left|a_{n}(z)\right|^{1 / n}$, and let $u^{*}(z)$ be the upper semicontinuous regularization of $u(z)$, i.e.,

$$
u^{*}(z)=\varlimsup_{z^{\prime} \rightarrow z} u\left(z^{\prime}\right)
$$

Then by the fact that $a_{n}(z)$ is locally uniformly bounded, we see that $u^{*}(z)$ is subharmonic on the disk $\Delta(0 ; 4)$, and it is easy to see that $u^{*}(z) \leq \frac{1}{6}$ for $|z|=3$. Hence by maximum principle we obtain that $u^{*}(z) \leq \frac{1}{6}$ for $|z| \leq 3$ and $u(z) \leq \frac{1}{6}$ for $|z| \leq 3$. It follows that the series (2.4) is holomorphic on $\left\{(z, w) \in \mathbf{C}^{2}|| z \mid<3\right.$ and $\left.|w|<6\right\}$. For the negative indices part we simply replace $w$ by $\frac{1}{w}$, then an analogous argument will go through as well. This completes the proof of the lemma.

It follows thus from Lemma 2.1 that $f_{k}(z, w) \in H(D)$ for $k=1,2$. We claim that in fact we have $f=\left(f_{1}, f_{2}\right) \in \operatorname{Aut}(D)$.

Proof of the claim. Put

$$
D_{0}=\left\{(z, w) \in \mathbf{C}^{2}|| z \mid<4 \text { and } 1<|w|<6\right\},
$$


and let $\widetilde{D}$ be the envelope of holomorphy of $D$. We have that $\Omega \subseteq D \subseteq \widetilde{D} \subseteq$ $D_{0}$. Let $p=\left(z_{0}, w_{0}\right)$ be a point in $D-\Omega$. Consider the circle

$$
C_{p}=\left\{\left(z, w_{0}\right) \in \Omega|| z \mid=3\right\} .
$$

By maximum modulus principle it is easy to see that $f(p) \in D_{0}$. We wish to show that $f(p) \in \widetilde{D}$, and hence $f(D) \subseteq \widetilde{D}$.

Suppose that $q=f(p) \notin \widetilde{D}$ with $5 \leq\left|f_{2}(p)\right| \leq 6$. Set

$$
E(\widetilde{D})=\widetilde{D} \cup\left\{(z, w) \in \mathbf{C}^{2}|| z \mid<4 \text { and }|w|<4\right\},
$$

and

$$
E_{+}(\widetilde{D})=\left\{(|z|,|w|) \in \mathbf{R}^{2} \mid(z, w) \in E(\widetilde{D})\right\} .
$$

It is well known that the logarithmic image of $E_{+}(\widetilde{D})$ is geometrically convex. Since the point $q^{*}=\left(\ln \left|f_{1}(p)\right|, \ln \left|f_{2}(p)\right|\right)$ is not in $\ln \left(E_{+}(\widetilde{D})\right)$ and the rational number is dense in $\mathbf{R}$, one can find two positive integers $m$ and $n$ such that the straight line $L$,

$$
L=\left\{(x, y) \in \mathbf{R}^{2} \mid m x+n y=c_{1} \text { for some constant } c_{1}\right\},
$$

go through $q^{*}$ and such that the whole set $\ln \left(\left|f\left(C_{p}\right)\right|_{+}\right)$lies in the half plane defined by $\left\{(x, y) \in \mathbf{R}^{2} \mid m x+n y-c_{1}<0\right\}$, where

$\left|f\left(C_{p}\right)\right|_{+}=\left\{(x, y) \in \mathbf{R}^{2} \mid(x, y)=\left(\left|f_{1}\left(z, w_{0}\right)\right|,\left|f_{2}\left(z, w_{0}\right)\right|\right)\right.$ for some $\left.|z|=3\right\}$.

Now consider the entire holomorphic function $h(z, w)=e^{-c_{1}} z^{m} w^{n}$. We see that the restriction of $h \circ f$ to $\Delta_{p}$, where $\Delta_{p}=\{(z, w) \in D|| z \mid<3$ and $\left.w=w_{0}\right\}$, will violate the maximum modulus principle. A similar argument via the mapping $w \mapsto \frac{1}{w}$ can be applied to show that no point of $D-\Omega$ can be mapped to $D_{0}-\widetilde{D}$ with $1 \leq|w| \leq 2$. This shows that $f(p) \in \widetilde{D}$, thus we have $f(D) \subseteq \widetilde{D}$.

Let $g$ be the inverse mapping of $f$. Since $g$ can be extended holomorphically to $\widetilde{D}$, it is legitimate to consider $g \circ f: D \rightarrow \mathbf{C}^{2}$. Then by identity theorem and the fact $\left.g \circ f\right|_{\Omega}=$ identity mapping, we get $g \circ f=$ identity mapping on $D$. Similarly $f \circ g$ is also the identity mapping on $D$. This shows that $f \in \operatorname{Aut}(D)$, and the proof of the claim is now completed.

Next we observe that the domain $D$ is Reinhardt. Therefore $f$ can be extended holomorphically to a small open neighborhood of $\bar{D}$. In particular, we have $f \in \operatorname{Aut}(\bar{D})$. For instance see Barrett [2]. However, we want to show more that $f$ in fact is given by a rotation in $w$-variable. So we next characterize a Reinhardt hypersurface in $\mathbf{C}^{2}$ that contains a Riemann surface in it. By a Reinhardt hypersurface we mean that the hypersurface is invariant under the rotations in all directions. The result might have some interest of itself. If $H$ is a Reinhardt hypersurface in $\mathbf{C}^{2}$, we denote by $H_{+}$the corresponding curve in $\mathbf{R}^{2}$. Then we have

Lemma 2.5. Let $H$ be a Reinhardt hypersurface in $\mathbf{C}^{2}$ such that $H_{+}$is decreasing in $\rho r$-space with $\rho=|w|$ and $r=|z|$. Then $H$ contains a Riemann surface near $p_{0} \in H$ if and only if $H$ is either flat in one of the coordinates or $H_{+}$is defined near $p_{0}$ by a hyperbola, namely,

$$
H_{+}=\left\{(\rho, r) \in \mathbf{R}^{2} \mid r \rho^{c}=\text { constant, for some } c \geq 0\right\} .
$$


Proof. Put $z=(x, y)=r e^{i \theta}$ and $w=(u, v)=\rho e^{i \phi}$. Let the defining function for $H_{+}$(hence for $H$ ) by $\xi(\rho, r)$. Rewrite $\frac{\partial}{\partial x}$ and $\frac{\partial}{\partial y}$ in terms of polar coordinates, we obtain

$$
\frac{\partial}{\partial x}=-\frac{\sin \theta}{r} \frac{\partial}{\partial \theta}+\cos \theta \frac{\partial}{\partial r}, \quad \frac{\partial}{\partial y}=\frac{\cos \theta}{r} \frac{\partial}{\partial \theta}+\sin \theta \frac{\partial}{\partial r} .
$$

Next the tangential type- $(1,0)$ vector field is generated by

$$
L=\frac{\partial \xi}{\partial z} \frac{\partial}{\partial w}-\frac{\partial \xi}{\partial w} \frac{\partial}{\partial z}=\frac{1}{2} e^{-i \theta} \frac{\partial \xi}{\partial r} \frac{\partial}{\partial w}-\frac{1}{2} e^{-i \phi} \frac{\partial \xi}{\partial \rho} \frac{\partial}{\partial z} .
$$

Suppose that $H$ contains a Riemann surface $\mathscr{R}$ near $p_{0}$ with $\frac{\partial \xi}{\partial r}\left(p_{0}\right) \neq 0$. Then one can choose $L$ to be

$$
L=\frac{\partial}{\partial w}-e^{i(\theta-\phi)} \cdot \frac{\partial \xi}{\partial \rho}\left(\frac{\partial \xi}{\partial r}\right)^{-1} \frac{\partial}{\partial z} .
$$

Put $g(z, w)=e^{i(\theta-\phi)} \cdot \frac{\partial \xi}{\partial \rho}\left(\frac{\partial \xi}{\partial r}\right)^{-1}$. Since $\left.[L, \bar{L}]\right|_{\mathscr{R}} \equiv 0 \bmod (L \oplus \bar{L})$, we see that the restriction $\left.g\right|_{\mathscr{R}}$ is holomorphic. Then consider

$$
\left.\frac{g w}{z}\right|_{\mathscr{R}}=\frac{\partial \xi / \partial \rho}{\partial \xi / \partial r} \cdot \frac{\rho}{r} \text {. }
$$

It shows that the function $\left.\frac{g w}{z}\right|_{\mathscr{R}}$ is holomorphic and real valued. Hence it must be a real constant function, namely,

$$
\left.\frac{g w}{z}\right|_{\mathscr{R}} \equiv c, \quad \text { for some } c \in \mathbf{R} \text {. }
$$

Also locally one can express $r$ as a function of $\rho$, and the slope of $H_{+}$near $p_{0}$ is given by

$$
\frac{d r}{d \rho}=-\frac{\partial \xi / \partial \rho}{\partial \xi / \partial r}=-c \frac{r}{\rho} .
$$

Therefore by solving this first order differential equation we get $r \rho^{c}=e^{c_{0}}$, for some constant $c_{0} \in \mathbf{R}$. Since $H_{+}$is decreasing, the constant $c$ is nonnegative.

On the other hand, if $H_{+}$is defined locally near some $p_{0}$ by $r \rho^{c}=c_{1}$ with $c, c_{1}>0$, then by direct computation we get

$$
L=w \frac{\partial}{\partial w}-c z \frac{\partial}{\partial z} .
$$

Hence we have $[L, \bar{L}] \equiv 0$. It follows that there exists a Riemann surface in $H$ near $p_{0}$. This completes the proof of the lemma.

It is interesting to note that the vector field $-2 \operatorname{Im} L$, where $L$ is given in (2.6), is generated by the following $S^{1}$-action,

$$
\begin{gathered}
\Lambda: S^{1} \times \mathscr{R} \rightarrow \mathscr{R}, \\
(\theta,(z, w)) \mapsto\left(e^{-i c \theta} z, e^{i \theta} w\right) .
\end{gathered}
$$

Now we go back to the automorphism $f$ of $D$. We see that $f$ will map biholomorphically a Riemann surface in the boundary onto another Riemann surface in the boundary. In particular, if we set

$$
\mathscr{R}_{a, b, \theta}=\left\{(z, w) \in b D \mid z=4 e^{i \theta} \text { and } a<|w|<b \text { with } a \leq 2 \text { and } b \geq 5\right\}
$$


to be the largest annulus sitting in the boundary with $|z|=4$, and set

$$
C_{a, \theta}=\left\{\left(4 e^{i \theta}, w\right) \in b D|| w \mid=a\right\}
$$

to be the inner boundary of $\mathscr{R}_{a, b, \theta}$, and similarly let $C_{b, \theta}$ be the outer boundary of $\mathscr{R}_{a, b, \theta}$. Then $f$ will map $\mathscr{R}_{a, b, \theta}$ to a Riemann surface in the boundary. We claim that $\mathscr{R}_{a, b, \theta}$ cannot be mapped to any Riemann surface contained in the boundary with $1<|w|<2$ or $5<|w|<6$. First it is not hard to see that there are only three different types of Riemann surfaces in these regions, they are

(i) $\mathscr{R}_{c} \subseteq\left\{\left.(z, w) \in b D|| z|| w\right|^{c}=A\right.$ for some constants $A$ and $c>0$, and $\alpha<|w|<\beta$ with $5<\alpha<\beta<6\}$, or an equivalent counterpart in the region $1<|w|<2$.

(ii) $\mathscr{R}_{z}=\left\{\left(z, w_{0}\right) \in b D \mid w_{0}=\rho e^{i \phi}\right.$ for some $\rho$ and $\phi$ with $5<\rho<6$ or $1<\rho<2$, and $s<|z|<t$ for some $3<s<t<4\}$.

(iii) $\mathscr{R}_{w}=\left\{\left(z_{0}, w\right) \in b D \mid z_{0}=r e^{i \theta}\right.$ for some $r$ and $\theta$ with $3<r<4$, and $s<|w|<t$ for some $1<s<t<2$ or $5<s<t<6\}$.

We can rule out $\mathscr{R}_{z}$ and $\mathscr{R}_{w}$ immediately by considering the ratio of the radii of the boundaries of these annuli. To knock out $\mathscr{R}_{c}$ we first observe that if $f$ maps $\mathscr{R}_{a, b, \theta}$ onto some $\mathscr{R}_{c}$, then by continuity $f$ must map $c_{b, \theta}$ for any $\theta$ into exactly one of $\{(z, w) \in b D|| w \mid=\alpha\}$ or $\{(z, w) \in b D|| w \mid=\beta\}$. Suppose that $C_{b, \theta}$ is mapped to $\{(z, w) \in b D|| w \mid=\alpha\}$. Then by maximum modulus principal we see that $f$ will map $\{(z, w) \in \bar{D}|| z \mid \leq 4$ and $|w|=b\}$ biholomorphically onto

$$
\left\{(z, w) \in \bar{D}|| z \mid \leq A / \alpha^{c} \text { and }|w|=\alpha\right\} .
$$

In particular, $f$ must map a disk

$$
\Delta_{w}=\left\{(z, w) \in D|| z \mid<4 \text { and } w=b e^{i \phi} \text { for some } \phi\right\}
$$

biholomorphically onto another disk

$$
\Delta_{w^{\prime}}=\left\{\left(z, w^{\prime}\right) \in D|| z \mid<A / \alpha^{c} \text { and } w^{\prime}=\alpha e^{i \phi^{\prime}} \text { for some } \phi^{\prime}\right\} .
$$

Since the restriction $\left.f\right|_{\Omega}$ is an automorphism, we also have the following biholomorphic equivalence between two annuli induced by $\left.f\right|_{\Omega}$, namely, $\left.f\right|_{\Omega}: \Delta_{w} \cap$ $\Omega \stackrel{\sim}{\rightarrow} \Delta_{w^{\prime}} \cap \Omega$. However, this cannot happen simply by examining the ratio of the radii of boundaries of these two annuli. Thus we have shown that $f\left(\mathscr{R}_{a, b, \theta}\right)=\mathscr{R}_{a, b, \eta(\theta)}$, for some real-valued function $\eta(\theta)$ that maps $S^{1}$ bijectively onto itself.

Next we divide our arguments into two subcases.

Case 1. If $f$ maps $C_{b, \theta}$ to $C_{b, \eta(\theta)}$ for some $\theta$. Then by continuity $f$ will map $C_{b, \theta}$ to $C_{b, \eta(\theta)}$ for all $\theta \in[0,2 \pi]$. For each fixed $\theta \in[0,2 \pi]$, we see by reflection principle that $f_{2}$ can be extended to an entire function which preserves the modulus on $|w|=a$ and $|w|=b$. Hence $f_{2}$ must take the following form

$$
f_{2}\left(4 e^{i \theta}, w\right)=e^{i \delta(\theta)} \cdot w
$$

for some real-valued function $\delta(\theta)$. Also we have

$$
f_{1}\left(4 e^{i \theta}, w\right)=4 e^{i \eta(\theta)},
$$

independent of $w$ for $a<|w|<b$. 
Then by the maximum modulus principle we have

$$
\left|f_{2}(z, w)\right|=|w| \text { for }|z| \leq 4 \text { and } a<|w|<b .
$$

This implies that $f$ will map $S_{c}$ biholomorphically onto $S_{c}$, where $S_{c}=$ $\{(z, w) \in \bar{D}|| z|\leq 4| w \mid,=c$ with $a<c<b\}$. Therefore, we conclude that $f$ must map a disk $\Delta_{c, \phi}$ onto another disk $\Delta_{c, \phi^{\prime}}$ i.e.,

$$
f: \Delta_{c, \phi} \stackrel{\sim}{\rightarrow} \Delta_{c, \phi^{\prime}},
$$

where $\Delta_{c, \phi}=\left\{(z, w) \in \bar{D}|| z \mid \leq 4, w=c e^{i \phi}\right\}$. Thus if we combine equations (2.7) and (2.9), we obtain for fixed $\phi$ that $f_{2}\left(4 e^{i \theta}, c e^{i \phi}\right)=c e^{i \delta(\theta)} \cdot e^{i \phi}=c e^{i \phi^{\prime}}$, for all $\theta \in[0,2 \pi]$. This implies that $\delta(\theta)$ is a constant function, namely, $\delta(\theta)=\phi_{0}$. Hence we obtain that $f_{2}(z, w)=e^{i \phi_{0}} w$.

Next equation (2.8) shows that the restriction $\left.f_{1}\right|_{\Delta_{c, \phi}}$ of $f_{1}$ to every disk $\Delta_{c, \phi}$ with $a<c<b$ and all $\phi$ has the same boundary value. So we conclude that $f_{1}(z, w)=f_{1}(z)$ is independent of $w$. Then by the facts that $\left|f_{1}\left(4 e^{i \theta}\right)\right|=4$ and $\left|f_{1}\left(3 e^{i \theta}\right)\right|=3$, we get

$$
f_{1}(z, w)=f_{1}(z)=e^{i \theta_{0}} z \quad \text { for some constant } \theta_{0} \in \mathbf{R} .
$$

Since $\left.f\right|_{\Omega}$ is an automorphism of $\Omega$, it is easy to see that $\theta_{0}=0$. This shows that

$$
f=\left(f_{1}, f_{2}\right)=\left(z, e^{i \phi_{0}} w\right),
$$

and the proof for Case 1 is now completed.

Finally we show that $f$ cannot map $C_{b, \theta}$ to $C_{a, \eta(\theta)}$. This will also complete the proof of our main theorem on the Barrett's domain.

Case 2. If $f$ maps $C_{b, \theta}$ to $C_{a, \eta(\theta)}$ for some $\theta$. Then again by continuity $f$ will map $C_{b, \theta}$ to $C_{a, \eta(\theta)}$ for all $\theta \in[0,2 \pi]$. Consider the map

$$
\begin{aligned}
g(z, w): D & \rightarrow D^{\prime}, \\
(z, w) & \mapsto\left(f_{1}(z, w), \frac{a b}{f_{2}(z, w)}\right),
\end{aligned}
$$

where $D^{\prime}$ is biholomorphic to $D$ via the map $(z, w) \mapsto\left(z, \frac{a b}{w}\right)$.

So one can repeat the above argument and obtain that

$$
f_{2}(z, w)=\frac{a b}{w} e^{i \phi_{0}} \quad \text { for some constant } \phi_{0} \in \mathbf{R} .
$$

Since $5 \leq a b \leq 12$, in order to preserve the boundaries at two ends, we must have $a b=6$. We may also conclude that

$$
f_{1}(z, w)=z \text {. }
$$

Next consider the point $p_{0}=\left(\frac{1}{2}, 3\right)$. We see that $p_{0} \in \Omega$. Since $f \in$ Aut $(\Omega)$, we must have $f\left(p_{0}\right) \in \Omega$. However, equations (2.11) and (2.12) show that $f\left(p_{0}\right)=\left(\frac{1}{2}, 2 e^{i \phi_{0}}\right)$, and this point is clearly not in $\Omega$. This gives the desired contradiction. 
Hence any automorphism on the Barrett's domain is given by a rotation in $w$-variable.

\section{Proof on the Diederich-Fornaess Domains}

We first recall briefly the definition of the Diederich-Fornaess domain here. Fix a smooth function $\lambda: \mathbf{R} \rightarrow \mathbf{R}$ satisfying

(a) $\lambda(x)=0$ if $x \leq 0$,

(b) $\lambda(x)>1$ if $x>1$,

(c) $\lambda^{\prime \prime}(x) \geq 100 \lambda^{\prime}(x)$ for all $x$,

(d) $\lambda^{\prime \prime}(x)>0$ if $x>0$,

(e) $\lambda^{\prime}(x)>100$ if $\lambda(x)>\frac{1}{2}$.

Then for any $r>1$ we define $\Omega_{r}=\left\{(z, w) \in \mathbf{C}^{2} \mid \rho_{r}(z, w)<0\right\}$ where $\rho_{r}(z, w)=\left|z+e^{i \ln |w|^{2}}\right|^{2}-1+\lambda\left(1 /|w|^{2}-1\right)+\lambda\left(|w|^{2}-r^{2}\right)$.

Theorem [8]. $\Omega_{r}$ is a smoothly bounded pseudoconvex domain in $\mathbf{C}^{2}$. The boundary is strictly pseudoconvex everywhere except on the following annulus,

$$
M_{r}=\left\{(z, w) \in b \Omega_{r} \mid z=0 \text { and } 1 \leq|w| \leq r\right\} .
$$

Now let $f=\left(f_{1}, f_{2}\right)$ be an automorphism of $\Omega_{r}$. Then $f$ can be extended smoothly up to the boundary on $b \Omega_{r}-M_{r}$. For instance, see Bell [5]. Therefore, if we consider the deleted torus

$$
T_{a}=\left\{(z, w) \in b \Omega_{r}|1<| w \mid=a<r \text { and } z \neq 0\right\},
$$

we see that $\eta_{r}=\rho_{r} \circ f$ is a defining function for $T_{a}, 1<a<r$, namely, the equation $\left|f_{1}(z, w)+e^{i \ln \left|f_{2}(z, w)\right|^{2}}\right|=1$ defines $T_{a}$. This implies that $\left|f_{2}(z, w)\right|^{2}=|w|^{2} \cdot e^{2 k \pi}$, for some fixed integer $k$. Hence by considering the points $(z, w) \in T_{a}$ with a close to either 1 or $r$, we conclude that $k=0$ and $\left|f_{2}(z, w)\right|=|w|$ for $(z, w) \in T_{a}$ with $1<a<r$.

Next fix the constant $a$ with $1<a<r$, and a point $z_{0}$ with $\left|z_{0}+e^{i \ln |a|^{2}}\right|<1$ such that $z_{0}$ lies in a small open neighborhood of $-2 e^{i \ln |a|^{2}}$. Then we consider the annulus defined by

$$
A_{z_{0}}=\left\{\left(z_{0}, w\right) \in \mathbf{C}^{2}\right\} \cap \Omega_{r}
$$

with the inner boundary $C_{\alpha}=\left\{\left(z_{0}, w\right) \in b \Omega_{r}|| w \mid=\alpha\right\}$ and the outer boundary $C_{\beta}$ such that $\alpha<a<\beta$. $A_{z_{0}}$ can be identified with $A=\{w \in \mathbf{C} \mid \alpha<$ $|w|<\beta\}$. Hence via this identification we obtain that

$$
f_{2}\left(z_{0}, C_{\alpha}\right)=C_{\alpha} \text { and } f_{2}\left(z_{0}, C_{\beta}\right)=C_{\beta},
$$

and $f_{2}\left(z_{0}, \cdot\right)$ can be extended to an entire function by reflection principle. Then by (3.1) we must have that

$$
f_{2}(z, w)=e^{i \phi(z)} \cdot w
$$

for some real-valued function $\phi(z)$. Since $f_{2}(z, w)$ is also holomorphic in $z$, we conclude that $\phi(z)=\phi_{0}$ for some constant $\phi_{0} \in \mathbf{R}$, and

$$
f_{2}(z, w)=e^{i \phi_{0}} \cdot w \quad \text { for }(z, w) \in \Omega_{r} .
$$

Then we consider the open solid torus $\pi_{a}$ defined by

$$
\pi_{a}=\left\{(z, w) \in \Omega_{r}|1<| w \mid=a<r \text { and }\left|z+e^{i \ln |w|^{2}}\right|<1\right\} .
$$


Put

$$
\Delta_{a, \phi}=\left\{\left(z, a e^{i \phi}\right) \in \Omega_{r}|| z+e^{i \ln |a|^{2}} \mid<1\right\} .
$$

It follows that the restriction of $f_{1}$ to $\Delta_{a, \phi_{1}}$ must map $\Delta_{a, \phi_{1}}$ biholomorphically onto $\Delta_{a, \phi_{2}}$ for some $\phi_{2}$. This implies that the restriction of $f_{1}$ to $\Delta_{a, \phi_{1}}$ can be extended at least smoothly up to $\overline{\Delta_{a, \phi_{1}}}$. Since $f_{1}\left(0, a e^{i \phi_{1}}\right)=0$, it follows that $f_{1}(z, w)$ can be expressed via the automorphisms on the unit disk as

$$
f_{1}(z, w)=e^{i \ln |w|^{2}}\left(\frac{1-\overline{b(w)} e^{i \ln |w|^{2}}}{e^{i \ln |w|^{2}}-b(w)}\right)\left(\frac{z+e^{i \ln |w|^{2}}-b(w)}{1-\overline{b(w)}\left(z+e^{i \ln |w|^{2}}\right)}\right)-e^{i \ln |w|^{2}}
$$

for some real analytic function $b(w)$ satisfying $|b(w)|<1$ for $1<|w|<r$. Equation (3.3) shows that there exists a small number $\varepsilon>0$ such that $f_{1}(z, w)$ is real analytic on $\Delta(0 ; \varepsilon) \times A_{\delta}$, where $A_{\delta}=\{w \in \mathbf{C}|1+\delta<| w \mid<r-\delta$ for some small $\delta>0\}$. This in turn implies that $f_{1}(z, w)$ is holomorphic on $\Delta(0 ; \varepsilon) \times A_{\delta}$. Therefore, one can write

$$
f_{1}(z, w)=\sum_{k=1}^{\infty} a_{k}(w) z^{k}
$$

with $a_{k}(w) \in H\left(A_{\delta}\right)$ for all $k \geq 1$. By direct computation we get

$$
a_{1}(w)=\frac{\partial f_{1}}{\partial z}(0, w)=\frac{1-|b(w)|^{2}}{\mid 1-\overline{b(w)} e^{\left.i \ln |w|^{2}\right|^{2}}} .
$$

It shows that $a_{1}(w)$ is a positive real constant, i.e., $a_{1}(w)=c>0$. Next the computation of $a_{2}(w)$ shows that

$$
a_{2}(w)=\frac{1}{2} \frac{\partial^{2} f_{1}}{\partial z^{2}}(0, w)=c \cdot \frac{\overline{b(w)}}{1-\overline{b(w)} e^{i \ln |w|^{2}}} .
$$

We claim that $a_{2}(w) \equiv 0$. Set

$$
g(w)=\frac{a_{2}(w)}{c}=\frac{\overline{b(w)}}{1-\overline{b(w)} e^{i \ln |w|^{2}}} \in H\left(A_{\delta}\right),
$$

we have

$$
\begin{aligned}
c & =\frac{1-|b(w)|^{2}}{\mid 1-\overline{b(w)} e^{\left.i \ln |w|^{2}\right|^{2}}}=\left|1+g(w) e^{i \ln |w|^{2}}\right|^{2}-|g(w)|^{2} \\
& =1+2 \operatorname{Re}\left(g(w) e^{i \ln |w|^{2}}\right) .
\end{aligned}
$$

Therefore, one can write

$$
g(w) e^{i \ln |w|^{2}}=c_{0}+i I(w)
$$

with $c_{0}=\frac{1}{2}(c-1)$ and $I(w)$ is a smooth real-valued function on $A_{\delta}$. Hence we obtain

$$
g(w)=c_{0} e^{-i \ln |w|^{2}}+i I(w) e^{-i \ln |w|^{2}} \in H\left(A_{\delta}\right) .
$$

Locally one can multiply equation $(3.5)$ by $e^{2 i \ln w}$ to get a new well-defined holomorphic function, and get

$$
g(w) e^{2 i \ln w}=c_{0} e^{-2 \operatorname{Arg} w}+i I(w) e^{-2 \operatorname{Arg} w} .
$$


The real part of $g(w) e^{2 i \ln w}$ is a harmonic function. So let $w=u+i v$, by direct computation we get

$$
\Delta_{w}\left(c_{0} e^{-2 \operatorname{Arg} w}\right)=c_{0} \Delta_{w}\left(e^{-2 \tan ^{-1} v / u}\right)=\frac{4 c_{0}}{u^{2}+v^{2}} e^{-2 \tan ^{-1} v / u} \equiv 0 .
$$

It follows that $c_{0}=0$, and hence $c=1$. This reduces $(3.5)$ to

$$
-i g(w)=I(w) e^{-i \ln |w|^{2}} \in H\left(A_{\delta}\right) .
$$

Then repeat the same argument, we see that

$$
-i g(w) e^{2 i \ln w}=I(w) e^{-2 \operatorname{Arg} w}=c_{1},
$$

where $c_{1}$ is a global constant. Hence

$$
I(w)=c_{1} e^{2 \operatorname{Arg} w}
$$

is a well-defined function on $A_{\delta}$. It forces $c_{1}=0$. This shows $g(w) \equiv 0$, and the proof of our claim is now completed.

It follows then from (3.4) that we have $b(w) \equiv 0$ on $A_{\delta}$, and equation (3.3) can be simplified to

$$
f_{1}(z, w)=z \text { on } \Omega_{r} \text {. }
$$

Our main theorem now follows from (3.2) and (3.8). So we are done.

\section{REFERENCES}

1. D. Barrett, Irregularity of the Bergman projection on a smooth bounded domain in $\mathbf{C}^{2}$, Ann. of Math. (2) 119 (1984), 249-255.

2. __ Holomorphic equivalence and proper mapping of bounded Reinhardt domains not containing the origin, Comment. Math. Helv. 59 (1984), 550-564.

3. __ Biholomorphic domains with inequivalent boundaries, Invent. Math. 85 (1986), 373377.

4. _ Behavior of the Bergman projection on the Diederich-Fornaess worm, Acta Math. 168 (1992), 1-10.

5. S. Bell, Local boundary behavior of proper holomorphic mappings, Proc. Sympos. Pure Math., vol. 41, Amer. Math. Soc., Providence, R. I., 1984, pp. 1-7.

6. S. Bell and E. Ligocka, A simplification and extension of Fefferman's theorem on biholomorphic mappings, Invent. Math. 57 (1980), 283-289.

7. H. P. Boas and E. J. Straube, The Bergman projection on Hartogs domains in $\mathbf{C}^{2}$, preprint.

8. K. Diederich and J. E. Fornaess, Pseudoconvex domains: an example with nontrivial Nebenhulle, Math. Ann. 225 (1977), 275-292.

Department of Mathematics, State University of New York at Albany, Albany, New YORK 12222 